sciendo

\title{
Naturalising Mathematics: \\ A Critical Look at the Quine-Maddy Debate
}

\author{
Marianna Antonutti Marfori
}

University of Bristol

Disputatio Vol. 4, No. 32

May 2012

DOI: 10.2478/disp-2012-0002

ISSN: 0873-626X 


\title{
Naturalising Mathematics: A Critical Look at the Quine-Maddy Debate
}

\author{
Marianna Antonutti Marfori \\ University of Bristol \\ BIBLID [0873-626X (2012) 32; pp. 323-342]
}

\begin{abstract}
This paper considers Maddy's strategy for naturalising mathematics in the context of Quine's scientific naturalism. The aim of this proposal is to account for the acceptability of mathematics on scientific grounds without committing to revisionism about mathematical practice entailed by the Quine-Putnam indispensability argument. It has been argued that Maddy's mathematical naturalism makes inconsistent assumptions on the role of mathematics in scientific explanations to the effect that it cannot distinguish mathematics from pseudo-science. I shall clarify Maddy's arguments and show that the objection can be overcome. I shall then reformulate a novel version of the objection and consider a possible answer, and I shall conclude that mathematical naturalism does not ultimately provide a viable strategy for accommodating an anti-revisionary stance on mathematics within a Quinean naturalist framework.
\end{abstract}

\section{Keywords}

Quinean scientific naturalism, confirmational holism, indispensability argument, revisionism, mathematical naturalism.

\section{Introduction $^{*}$}

In Second Philosophy. A Naturalistic Method (2007), Penelope Maddy puts forward a naturalised account of mathematics which purports to

* Earlier versions of this paper were presented at the conferences Word and $\mathrm{Ob}$ ject, 50 Years Later and SIFA 2010. I thank both audiences - and particularly Jacob Busch, Sthatis Psillos and Andrea Sereni - for interesting questions. Many thanks to Leon Horsten and two anonymous referees for this Journal for helpful comments. I gratefully acknowledge the financial support of the Department of Philosophy of the University of Bristol.

Disputatio, Vol. IV, No. 32, May 2012 
do justice to the special status of mathematics while maintaining a Quinean naturalist framework.

Quinean Scientific Naturalism can be characterised as the thesis that science is not answerable to any extra-scientific tribunal and is the ultimate arbiter of truth. In Quine's words, 'it is within science itself, and not in some prior philosophy, that reality is to be identified and described' (1981: 21). According to Quinean scientific naturalism, mathematics is part of our overall theory of the world and, as such, is continuous with science both on the ontological and methodological level; our best scientific theories dictate both what we should include in our ontology, and what are the legitimate ways of enquiring into the natural world.

Quinean naturalists are committed to the so-called Quine-Putnam indispensability argument, according to which we ought to commit to the existence of all and only those entities that are indispensable to our best scientific theories. A significant consequence of this view for mathematics is that only those bits of mathematics which actually figure in our best scientific theories should be regarded as justified. This consequence is taken to be problematic because it implies a revisionary stance towards mathematical practice. That is, it implies that certain aspects of mathematical practice, namely those that do not (aim to) contribute to our best scientific theories, are not legitimate.

Such an undesirable consequence is what motivates Maddy to reject the indispensability argument and to propose a novel strategy for the naturalisation of mathematics. Maddy's naturalism is a particularly attractive position because it seems to constitute the only option available to a Quinean naturalist who is uncomfortable with both revisionism about mathematical practice and a nominalist stance on mathematics. The guiding strategy of Maddy's mathematical naturalist is to avoid the commitment to revisionism by rejecting the conclusion of the indispensability argument, while at the same time maintaining that mathematics plays a crucial role in our understanding of the world because it is indispensable to scientific practice.

The aim of this paper is to assess whether there is a viable way of combining Quinean scientific naturalism with Maddy's nonrevisionary stance towards mathematical practice. The structure of the paper is the following. In the first section I present the standard version of the indispensability argument and Quine's revisionism about mathematical practice, and make a few remarks on some mat- 
ters of interest in the context of the present discussion. I then present Maddy's own view on the indispensability argument and her solution to avoid revisionism. Her position is subtle, so it is important to consider Maddy's objections to the indispensability argument, and her motivations for her positive view of mathematics, in detail. In the third section an important objection against mathematical naturalism made by Dieterle (1999) is considered, and it is argued that the objection is not conclusive. In the following section I outline Maddy's reply and show how it could successfully counter Dieterle's objection if cashed out in more detail. The analysis will suggest that Maddy's reply crucially relies on indispensability considerations but leaves the appeal to indispensability unargued, so I shall formulate a new objection which is more charitable to mathematical naturalism. Finally, in section five, I shall outline a possible response on behalf of the mathematical naturalist and I shall argue that such a strategy is not available to the mathematical naturalist due to an underlying tension in Maddy's position. I shall conclude that this tension leads to a dilemma that forces the mathematical naturalist to either embrace Quinean scientific naturalism along with a revisionary stance on mathematical practice, or to accept the undesirable consequence of mathematics being indistinguishable from pseudo-science. Either way, the moral to be drawn is that Maddy's mathematical naturalism is not a viable way of combining anti-revisionism about mathematical practice and Quinean scientific naturalism.

\section{Naturalism and Indispensability}

A standard characterisation of the indispensability argument is given by Colyvan (2001):

1. We ought to have ontological commitment to all and only those entities that are indispensable to our best scientific theories

2. Mathematical entities are indispensable to our best scientific theories

Therefore, 
3. We ought to have ontological commitment to mathematical entities.

The thesis that we ought to commit to the truth of the statements that feature in our best scientific theories taken at face value (premise1) is scientific realism. In a Quinean framework, scientific realism results from the conjunction of (i) Quinean scientific naturalism (the thesis that science is the ultimate arbiter of truth) and (ii) confirmational holism (the thesis that scientific theories face the tribunal of experience as a whole).

The indispensability argument is considered the strongest naturalistic argument for mathematical realism (see e.g. Field 1980: 5). If sound, it allows us to draw ontological conclusions from the successful application of mathematics in the empirical sciences to the confirmation of the existence of mathematical entities. Indeed mathematical entities, on Quine's view, are on a par with theoretical entities in scientific theories: since there is no non-arbitrary way of discerning the support conferred by empirical evidence to theoretical entities referred to in scientific theories, and mathematical and theoretical entities are equally indispensable to scientific theories, it follows that mathematical entities are empirically confirmed in the same way as theoretical entities are (see Quine 1969: 97-98 and 1981:149-151).

Analogously, on this view, mathematics and science are also epistemologically on a par: when a scientific theory is confirmed, the mathematics which is required in the formulation of the theory also gets confirmed. More precisely, the soundness of each methodology employed in scientific practice is empirically tested along with the existence of the theoretical entities referred to in those theories. Scientific method as a whole determines what further methodologies are accepted as legitimate in our pursuit of knowledge of the world and what justificatory standards are authoritative in adjudicating among competing theories. Since mathematical methods are indispensable to scientific practice, the Quinean naturalist takes them to be legitimate tools in our pursuit of knowledge.

Maddy is a Quinean scientific naturalist insofar as she thinks that existence questions are ultimately settled by science and that scientific method is the most fundamental justification for determining what exists. However, she notices that in mathematical and scientific practice the questions about the existence of mathematical entities are not meant to be questions about the physical existence of those entities. Scientists are neither concerned about identifying the sort of 
empirical evidence that would confirm the existence of mathematical entities (such as e.g. real numbers), nor are they concerned about the lack of confirmation of their existence (2007: 317). Conversely, it could be argued that mathematicians are not driven by scientific method in adjudicating questions about mathematical existence. Considerations about what mathematical objects there are, and such objects' properties, are typically settled by mathematical methods. On the basis of these considerations, questions about ontological commitment and proper method can be tackled separately. ${ }^{1}$ Maddy recommends neutrality with respect to mathematical ontology, and focuses on methodological questions such as what makes for an acceptable axiom, a dependable method of proof, and so on. ${ }^{2}$ Accordingly in this paper I shall follow Maddy's concern for methodological issues.

Given this focus on methodological issues, I reformulate the indispensability argument as follows:

1'a. We ought to commit to the truth of all and only those theoretical hypotheses that are confirmed according to our best scientific theories' standards of confirmation (Quinean scientific naturalism)

1 'b. Scientific statements are not confirmed individually, but only as a corporate body (confirmational holism)

2'. Mathematics is indispensable to our best scientific theories

Therefore,

3'. We ought to commit to the truth of mathematical statements.

Our best scientific theories jointly constitute our best overall theory of the world. The Quinean scientific naturalist commits to the thesis that we are justified in believing an overall theory of the world

${ }^{1}$ In particular, the epistemological question of what justificatory standards are authoritative in mathematics becomes primary once we deny confirmational holism; more on this will be said in the next section.

${ }^{2}$ Questions about justificatory standards are both epistemological and methodological questions, so I shall use the terms interchangeably when talking about issues concerning justification of mathematical statements. 
to the extent that it is sanctioned by our current scientific standards, and earns the conclusion that we are justified in believing indispensable mathematics to be true. The mathematical naturalist holds that mathematics is to be accepted insofar as it is part of our best overall theory of the world, but denies premise 1'b of the argument. If confirmation does not holistically extend among different components of scientific theories, 1'a does not entail that mathematical statements are to be believed literally true of the world. So the mathematical naturalist is committed to believe all empirically confirmed scientific statements to be true, but does not have to commit to the claim that only empirically confirmed statements are to be accepted. ${ }^{3}$ Thus the mathematical naturalist endorses a weaker version of scientific realism, according to which we ought to accept (as opposed to believe to be true) all (as opposed to all and only) those theoretical hypotheses that are confirmed according to our best scientific theories' standards of confirmation.

To support the view that a good (or the best, as the naturalist would rather say) overall theory of the world is one which is at least compatible with our scientific standards, with the view that scientific theories are to be believed on the basis of their being part of our best overall theory of the world is obviously circular. However, there is shared agreement on the thesis that naturalism is not a philosophical doctrine which can be established by means of compelling arguments, but rather a meta-philosophical attitude which can only be recommended. In this sense, this kind of circularity does not represent a dangerous worry for the scientific and mathematical naturalist alike insofar as it is already implicit in the naturalistic core tenet commending philosophical modesty (see Maddy 2007: 235).

\section{Indispensability and Revisionism}

Maddy wants to block the problematic consequence of the indispensability argument that only the part of mathematics which is applied in natural science has a legitimate epistemological status. She does that by rejecting confirmational holism (premise 1 'b) on the basis of a

\footnotetext{
${ }^{3}$ See $\S 2$ for an account of why the denial of confirmational holism allows the mathematical naturalist to hold different epistemic attitudes towards different components of scientific theories.
} 
historical analysis of scientific practice. According to Maddy, such analysis shows that scientific theories are not regarded as a homogeneous whole up for confirmation as a unit, but that

The mere presence, even indispensable presence, of a posit in our theory of the world is not enough to warrant the conclusion that its existence has been established. ... [T]he mathematical posits appear in descriptions that we don't regard as true, from which it would be inappropriate to draw ontological morals of any kind (2007: 315-316). ${ }^{4}$

For the sake of the argument, I shall assume that confirmational holism can be successfully denied.

The indispensability argument crucially relies on confirmational holism for the inference from the empirical confirmation gained by successful scientific theories to the confirmation of the bits of mathematics which are indispensable to formulate those theories. If confirmation does not holistically extend among different theoretical components of any scientific theories, it also does not extend from any scientific theory to a mathematical theory indispensable to its formulation. Therefore, if confirmational holism is denied, we are not entitled to draw ontological and epistemological conclusions about mathematics on the grounds of successful application of (part of) mathematics to natural science. Furthermore, we are allowed to have different epistemic attitudes towards different components of our theory of the world and, in particular, about the empirical and mathematical components of a theory. Hence, the revisionary stance on mathematical practice can be avoided.

Maddy's rejection of the indispensability argument for mathematics parallels her rejection of confirmational holism for science. In both cases the grounds for rejection are the misrepresentation of the actual practice of science and mathematics offered in the corresponding argument, where an accurate account of scientific and mathematical practice are the primary concern of the naturalist. In particular, she argues that scientific practice, contra confirmational holism, shows that scientists help themselves to whatever mathematics best suits their purposes. They are not concerned about the ontological status of

\footnotetext{
${ }^{4}$ The historical case study that Maddy analyses is the gradual acceptance of the existence of atoms; for an extensive discussion of Quinean holism see Maddy (2007; I.6; IV.2.i).
} 
the theoretical entities involved, and simply regard mathematical statements either as useful tools or even as literally false idealisations (ibid.). In the same way, contra the indispensability argument, mathematicians do not see their activity as constrained or even guided by the necessity of application (2007: 345). The grounds for believing theorems are deduction from axioms, appeal to mathematical intuition, or other intra-mathematical considerations. Such intramathematical considerations drive mathematicians' pursuit of novel, pure mathematical theories. ${ }^{5}$ So just as Maddy's criticism to confirmational holism points at an implicit tension between the holistic principle - that scientific theories are up for confirmation as a whole - and actual scientific practice, her criticism to the indispensability argument points at an implicit tension between the claim that only applied mathematics matters and actual mathematical practice. That is, the indispensability argument portrays mathematical practice in a misleading way by claiming that only empirically confirmed parts of mathematics are granted a legitimate epistemological status. Thus, ultimately, the indispensability argument should be rejected on naturalistic grounds.

A truly naturalistic attitude towards mathematical practice, on Maddy's view, also recommends the naturalist philosopher not to be revisionary about mathematical practice itself. If the commitment to indispensable mathematics had been the prevailing guiding principle of scientific and mathematical practice, many mathematical theories initially pursued for purely mathematical reasons, but later shown to be of fundamental importance for natural science - would not have been developed (see e.g. the case of group theory for quantum mechanics, 2007: 347). A careful historical analysis of the practices involved shows that mathematics can best serve the needs of science when left flourishing without any extra-mathematical constraints. Therefore the mathematical naturalist is not entitled to reject some parts of accepted mathematical practice, which do not feature in scientific explanations, sanctioning only those parts of mathematics that receive empirical confirmation in virtue of their indispensability to successful scientific theories.

\footnotetext{
${ }^{5}$ By pure mathematics Maddy means 'mathematics pursued for its own reasons, using its own methods, quite independent of [the naturalist's] well-honed arsenal of observation, experiment, theory formation and so on', and 'away from the necessity of application' (2007: 345).
} 
A question may arise as to what extent confirmational holism is in fact revisionary about mathematical practice. In his reply to Parsons, Quine (1986: 400) suggests that the scientific naturalist is not compelled to regard only strictly indispensable mathematics as confirmed, but can consider as confirmed whatever mathematics is required for 'rounding out' indispensable mathematics. On this account, e.g., much of set theory would turn out to be included in confirmed mathematics in virtue of the fact that it underwrites most contemporary mathematics (see also Colyvan 2007), and only higher reaches of set theory would be considered as purely recreational. Ultimately, a very small fragment of mathematics is so isolated from the rest that it does not appear at any point of a chain of applications which bottoms out with applications in empirical science. So the price to pay in terms of methodological autonomy of mathematics for endorsing confirmational holism is after all not high. If confirmational holism is not revisionary in a substantive sense, the mathematical naturalist may not be able to reject the indispensability argument on naturalistic grounds.

However, Maddy's mathematical naturalism is not affected by this move. Even if indispensable mathematics is extended to include most of pure mathematics, Maddy's concern is precisely to argue that mathematics as a whole has a legitimate epistemological status. This includes absolutely unapplied mathematics, such as the higher reaches of set theory, one of Maddy's primary concerns in both her 1997 and 2007. So regardless of how revisionary the indispensability argument is, the mathematical naturalist would reject it on the grounds that mathematical practice is not conducted as if the indispensability argument was true.

Such considerations motivate Maddy to depart from Quinean scientific naturalism and reformulate the naturalist's meta-philosophical attitude towards mathematics. As an extremely successful enterprise, mathematics should be understood as it is practiced. Again, a good starting point would be looking at the history of mathematics. This reveals that until the eighteenth century mathematics was entrenched with science and philosophy. From the nineteenth century mathematics started differentiating its standards and goals from those of the other practices, freed itself from the purposes of serving science, and claimed its autonomy in pursuing purely mathematical problems by means of purely mathematical methods. Thus, according to Maddy, the naturalistic philosopher should, on naturalistic grounds, grant to 
mathematics the same respect that Quinean scientific naturalism grants to science. Mathematics should be understood and evaluated on its own terms and according to its own standards. The methodological autonomy of mathematics is the core of Maddy's mathematical naturalism:

Mathematics should be understood and evaluated in its own terms, and should not be subject to criticism from, and does not stand in need of support from, some external, supposedly higher point of view (be it scientific or philosophical). ${ }^{6}$

\section{Astrological and Theological Naturalism}

While maintaining Quinean scientific naturalism with respect to science, the naturalistic philosopher à la Maddy has three important advantages over the naturalistic philosopher à la Quine. Firstly, in virtue of her rejection of confirmational holism she can consistently avoid adopting a revisionary stance towards the methodology of currently accepted mathematical practice. Secondly, for the same reason, she can also be a scientific naturalist without being committed to the literal truth of every statement that features our best overall theory of the world. Thirdly, she can account for the special status of mathematical methodology with respect to scientific methodological standards.

It is not clear, however, that the methodological autonomy of mathematics comes at a lesser price than its curtailment in the light of experimental evidence. Mathematical naturalism seems to yield the problematic consequence that if mathematical justificatory standards are independent from scientific standards of confirmation, mathematics is on a par with lots of other intellectual enterprises whose results we would count as pseudo-science. For example, it may be argued

\footnotetext{
${ }^{6}$ The thesis as presented here is to be found in Maddy (1997:184). In 2007, Maddy modifies and reformulates many aspects of her 1997 view (including a substantial shift in terminology, from mathematical naturalism to second philosophy of mathematics) but maintains the core tenets. For ease of exposition, I shall here stick to the original terminology but shall refer to her recent work (Maddy 2007) unless otherwise stated.

The plausibility of mathematical naturalism arguably relies on the methodological unity of mathematics, which is itself a highly problematic issue. However, for reasons of space, I shall not consider this point here.
} 
that astrology is such an enterprise: it has its own methodological standards and ontological commitments which are assessed and justified, as in the case of mathematics, on the basis of its own standards.

Maddy's reply to this objection is in line with Quinean scientific naturalism. For astrology makes causal claims about spatio-temporal reality, the naturalistic philosopher would simply treat astrological claims as scientific claims and test them against scientific method, eventually showing that they are not adequately supported by empirical evidence (1997: 203-5, 2007: 107-9). However, even though scientific testing undermines astrological empirical predictions it tells us nothing about the status of mathematics. The mathematical naturalist, unlike the Quinean naturalist, would not consider mathematical statements as saying anything about the spatio-temporal realm because of the way the practitioners themselves regard mathematical statements in their ordinary practice, and she would not consider any mathematical statements as up for confirmation by scientific testing.

Maddy acknowledges that the same worry may be reformulated at a more abstract level to include the case of not empirically testable disciplines. For example, we may think of a discipline, pure theology, not making any causal claims but describing the interactions of God and angels in an abstract realm (2007: 346). As pure mathematics, this discipline would not straightforwardly be a part of science, though it would be in principle liable to figure in scientific explanations. This discipline would be in all relevant respects analogous to pure mathematics, including its methodological autonomy and unrevisability in the light of scientific standards. ${ }^{7}$ In this case the mathematical naturalist has apparently no grounds for rejecting theological naturalism as unscientific.

Dieterle (1999) argued that the mathematical naturalist cannot meet this objection because the argument in support of mathematical

${ }^{7}$ The idea that mathematics is not counterfactually bound to states of affairs in the world is implicit throughout all of Maddy's discourse. Though in the context of mathematical naturalism the unrevisability of mathematics cannot be explained by appealing to the centrality of mathematics in the web of beliefs as in Quinean scientific naturalism, Maddy never explicitly defends this claim nor considers why even applied mathematics would be unrevisable on scientific grounds (see also Tennant 2000). For reasons of space, I shall not address this issue here. For a discussion of the debate over the question whether Quinean scientific naturalism holds mathematics to be on a par with other parts of scientific theories in terms of confirmation but not in terms of falsification, see Busch forthcoming. 
naturalism ultimately rests on indispensability considerations, and without the appeal to indispensability the Quinean naturalist cannot reject astrological or theological naturalism as unscientific.

More specifically, Dieterle argues that if mathematics was just a tool for scientists (as Maddy seems to suggest), then unapplied mathematics and theology are on a par and do not figure in scientific explanations, and the Quinean scientific naturalist only has to accept those parts of mathematics that are applied. This view, however, just is Quinean scientific naturalism. If, on the other hand, mathematics is more than a mere tool, then the fact that mathematical statements figure in scientific explanations means that mathematical statements say something about the spatio-temporal realm. If it was so, then applied mathematics would be on a par with astrology and pure mathematics would be on a par with pure theology. The reason for the first analogy is that both applied mathematics and astrology would make causal claims about the spatio-temporal realm, but would be equally unrevisable in the light of scientific standards. In the second case, mathematical and theological statements would be equally acausal but liable to figure in scientific explanations. Therefore

'Maddy's use of indispensability considerations in the defence of mathematical naturalism ultimately either (1) undermines mathematical naturalism itself, leaving us with only scientific naturalism, or (2) leaves open the possibility of other unpalatable naturalisms' (1999: 131).

Despite representing an important threat to mathematical naturalism, this objection is not conclusive. Indeed, it takes Quinean scientific naturalism as the background view in line with Maddy, but implicitly relies on confirmational holism - a thesis that, as seen before, the mathematical naturalist can consistently deny. This allows the mathematical naturalist to further deny that indispensably figuring in scientific explanations entails empirical confirmation. Thus, the mathematical naturalist is entitled to grant mathematics methodological autonomy and a legitimate epistemological status without committing her to the ontological and epistemological consequences of the indispensability argument.

In the next section I shall analyse the mathematical naturalist's grounds for a response to Dieterle's objection. I shall then articulate a further worry also originating from the appeal to indispensability 
considerations and, in the final section, I shall outline and assess a possible argument in support of mathematical naturalism.

\section{Maddy's reply}

Maddy denies that applied mathematics has a different epistemological status from pure mathematics just in virtue of the former's successful application to science. However, this does not amount to having established that mathematics as a whole has a legitimate epistemological status independently of scientific standards. According to Maddy, it is possible to ground the legitimate epistemological status of mathematics as a whole in the role that mathematics plays in our overall theory of the world and in its importance in our pursuit of truth.

To support this point, Maddy considers the following thought experiment. Though providing tools for natural science is not the primary aim of pure mathematics, it is still one of its main aims. If mathematicians decided to pursue utterly different goals, Maddy contends, then mathematics would become irrelevant to science, and science would have to replace it with a different tool, say mathematics*. So if old mathematics was like pure theology its role would also be similar, and the new tool, mathematics*, would play the role that mathematics played before in the naturalistic philosopher's investigation (2007: 150-151; 2007: 350). If, conversely, parts of pure theology were discovered to be applicable, then pure theology would be functioning as the old pure mathematics, and it would have the same role the mathematical naturalist grants to actual pure mathematics.

The thought experiment purports to suggest that what confers a legitimate epistemological status to mathematics is not its methodological autonomy, but its indispensability as a tool in the pursuit of our understanding of the world. Thus the objection that the mathematical naturalist is compelled to deny either the usefulness of mathematics or its methodological autonomy can be resisted by appealing to the place that mathematics occupies in our overall theory of the world.

More generally, on this view, what distinguishes mathematics from science is its methodological solipsism; pure mathematics develops by pursuing purely mathematical goals, and is not subject to revisability in the light of scientific standards of confirmation. On the 
other hand, what distinguishes mathematics from other forms of nonscientific enquiry is not its methodological solipsism, but the indispensable role that it plays in our overall theory of the world. However, is the indispensability of mathematics a consideration that the mathematical naturalist can legitimately appeal to in order to discern mathematics from pseudo-science?

Again, Maddy does not explicitly argue in support of this move, but an argument on behalf of the mathematical naturalist could be outlined in the following way. Quinean scientific naturalism holds that we are justified in accepting an overall theory of the world to the extent that it is confirmed by empirical evidence. Accordingly, we ought to accept any theory which is part of our best overall theory of the world, whereby such a theory is recognised as scientific. As mathematics is indispensable to scientific theories, it is also indispensable to our best overall theory of the world (the indispensability relation being transitive). Hence, we ought to accept mathematics.

This argument seems to run into an obvious objection, again targeted at the appeal to indispensability considerations. Because of her rejection of the indispensability argument, the mathematical naturalist cannot appeal to the application of mathematics in natural science in order to ground the acceptance of mathematics (call this the applicability objection). ${ }^{8}$ Maddy is aware of this objection:

[The appeal to the usefulness of mathematics to natural science] is not a reversion to a Quinean indispensability argument, because the conclusion is only that mathematics is different from pure astrology, not that mathematics is confirmed (2007: 346, footnote 4).

In other words, what is being denied by the mathematical naturalist is not the claim that mathematics is indispensable to scientific practice, but the inference from the indispensable role that applied mathematics plays in scientific explanations to the confirmation of the existence of mathematical entities and the legitimacy of applied mathematical methods. The mathematical naturalist can recognise the autonomy of mathematics with respect to scientific practice while not denying its crucial role within our best overall theory of the world. This crucial role in fact makes mathematics as a whole different from pseudo-

\footnotetext{
${ }^{8}$ Maddy uses the terms indispensability and usefulness of mathematics with respects to scientific practice interchangeably, so I shall here conform to her use.
} 
science, and not - as the indispensability argument purports to establish - the indirect empirical confirmation received by some mathematical statements through their successful application in scientific practice.

\section{How Indispensable is the Indispensability Argument?}

Despite the appeal of this proposal, I shall argue that the strategy outlined above is unavailable to the mathematical naturalist. If the argument is correct, then mathematical naturalism cannot meet the applicability objection and thus ultimately fails to explain the difference between mathematics and pseudo-science.

It is important to notice that the criterion for discerning the success of any practice is ultimately scientific, for both the scientific and the mathematical naturalist. The success of a practice is determined by the role it plays in our best overall theory of the world. Since (in line with Quinean scientific naturalism) our best overall theory of the world is adjudicated by our current scientific standards, it trivially follows that any practice that plays a crucial role in our current best overall theory of the world is ultimately accepted on the grounds of the very same standards. As seen before, astrology is dismissed as pseudo-science by empirically testing its causal claims, and pure theology by observing that it does not play any role in our best overall theory of the world.

Only one part of mathematics, however, is involved in scientific explanations and empirical predictions. So there is an asymmetry in the standards of acceptance for pure and applied mathematics: applied mathematical methodology is both accepted on scientific grounds and sanctioned by mathematical standards, whereas pure mathematical methodology is just sanctioned by mathematical standards. Mathematical naturalism does not warrant the acceptance of pure mathematics by scientific standards. ${ }^{9}$ In order to conclude that mathematics

${ }^{9}$ It follows from this that the mathematical naturalist cannot even claim that mathematics is a successful enterprise as a whole simply on the basis of historical study and careful observation of mathematical practice. The criterion for a practice to be successful is still a scientific one, and pure mathematics cannot be judged successful on scientific grounds in the absence of a positive argument in support of this claim. So the mathematical naturalist can only claim that mathematics is a successful enterprise as a whole conditionally upon the soundness of Maddy's reply to the applicability objection. 
as a whole ought to be accepted, the mathematical naturalist must be able to warrant the inference from the usefulness of a part of mathematics to the acceptance of mathematics as a whole. Therefore, some sort of holistic extension of acceptance is needed in order to establish the conclusion that pure mathematical standards ought to be accepted on the basis of scientific standards (i.e., that mathematics as a whole is accepted on scientific grounds) because of the successful application that part of mathematics receives in scientific practice.

At this point the mathematical naturalist may decide to bite the bullet and support this strategy, still without committing to confirmational holism. She has in fact strong reasons not to accept the ontological consequences of the indispensability argument - the consequences violate the naturalistic maxim not to be revisionary about a successful practice. She has, however, no principled reasons against a weaker version of holism which only focuses on the grounds for acceptability of scientific methodologies, and which respects the tenets of mathematical naturalism with respect to ontological matters. The idea of a methodological holism may be formulated as follows: the soundness of the methodological maxims employed in our pursuit of knowledge of the external world is not tested individually, but methodologies as a whole receive epistemological legitimacy through successful application in scientific practice. Given the central tenet of mathematical naturalism, according to which mathematical methodology should not be subject to extra-mathematical criticism, if the mathematical naturalist assumes methodological holism, then for pure mathematics to be part of our best overall theory of the world just is for it to be sanctioned by its own methods and standards. On the assumptions that, firstly, a theory is recognised as scientific if it is part of our best overall theory of the world, and that, secondly, mathematics is indispensable to our best overall theory of the world, we can grant mathematics as a whole the status of a scientific theory even though it is sanctioned by its own methods and standards.

By appealing to methodological holism the mathematical naturalist can draw the desired epistemological conclusion that mathematical methodology ought to be accepted as a whole. This follows from the vindication of the methodology of applied mathematics through the indispensable role of applied mathematics in science. She can thus maintain that the credibility of mathematics rests on a scientific basis even though mathematics is not subject to scientific criticism. This provides the mathematical naturalist with the desired criterion to 
distinguish pure mathematical methodology from pseudo-scientific methodologies on the basis of the indispensable role that mathematics as a whole plays in our best overall theory of the world. Furthermore, not only does methodological holism respect the ontological neutrality of the mathematical naturalist, but it also justifies the acceptance of mathematics regardless of any stance a naturalist may have with respect to mathematical ontology. Methodological holism allows the naturalist to remain faithful to Quinean scientific naturalism for what concerns ultimate matters of existence of mathematical entities, but at the same time allows her to vindicate the methodological autonomy of mathematics and its special epistemological status among sciences.

However, is methodological holism a viable option for the mathematical naturalist? Since the naturalistic standards for acceptance are scientific, the extension of the acceptability from applied mathematical methodology to pure mathematical methodology is only warranted on the grounds of a possible future application of pure mathematical methods in scientific practice. As such, adopting methodological holism seems to be in tension with the mathematical naturalistic prescription not to take applied mathematical methodology as a guide to mathematical methodology as a whole. Yet, granting epistemic value to pure mathematics regardless of any possible application is precisely the motivation behind mathematical naturalism. As briefly seen above, Maddy argues that the history of mathematics shows how from the nineteenth century mathematics started differentiating its standards and goals from those of other scientific practices, and providing useful tools for scientific practice ceased to be the main concern of mathematicians. If it had not been so, many mathematical theories initially pursued out of purely mathematical interest and devoid of any application would not have been developed. Hence, endorsing methodological holism would result in the failure to appreciate the methodological autonomy of mathematics, and in the adoption of a revisionary stance towards pure mathematical practice, which the mathematical naturalist forcefully rejects.

In summary, the claim that mathematics as a whole is acceptable on scientific grounds only follows if methodological holism is assumed, but methodological holism cannot be assumed because it is incompatible with the mathematical naturalistic prescription not to be revisionary about mathematical practice. Therefore the mathematical naturalist faces a dilemma: either she accepts methodological holism at the price of a revisionary stance on mathematical practice, or she 
rejects methodological holism at the price of losing a criterion to distinguish pure mathematics from pseudo-science.

More specifically, we have seen that the assumption of methodological holism allows the mathematical naturalist to (i) justify pure mathematical methodology on scientific grounds, and (ii) to distinguish pure mathematics from pseudo-science. However, methodological holism commits the mathematical naturalist to making scientific standards authoritative in the assessment of mathematical methodology, in line with Quinean scientific naturalism. This ultimately forces the mathematical naturalist to deny her maxim that mathematics should not be subject to extra-mathematical standards, and to deny the scientific status of pure mathematics, resulting in the adoption of a revisionary stance on mathematical practice. Thus if the mathematical naturalist accepts methodological holism, her position will be indistinguishable from Quinean scientific naturalism.

On the other hand, if the mathematical naturalist does not give up on the methodological solipsism of mathematics but rejects methodological holism, she cannot appeal to the indispensable role that mathematics plays in our overall theory of the world to justify the scientific status of pure mathematics. Without an holistic extension of acceptance from applied to pure mathematical methodology, pure mathematics is only sanctioned by mathematical standards. This makes mathematics completely devoid of application not indispensable to science, and accordingly not a discipline whose methodology is to be included among the methodologies of the scientific theories which constitute our best overall theory of the world. Thus if the mathematical naturalist rejects methodological holism, she is left with no criterion to discern pure mathematical methodology from pseudoscientific ones. Therefore, she has to conclude that pure mathematics and pure theology are methodologically on a par, and she has to grant theological naturalism the same respect that she grants mathematical naturalism.

\section{Conclusions}

In this paper, I have considered one of the most influential contemporary views on the naturalisation of mathematics and I have argued that despite its advantages, it ultimately falls short of accounting for the special status of mathematical practice within the framework of 
Quinean scientific naturalism. I considered an important objection to mathematical naturalism which focuses on the appeal to the usefulness of mathematics and showed that the mathematical naturalist can resist it because of her rejection of confirmational holism. I then reformulated the worry by taking into account Maddy's (2007) reply, and argued that her rejection of confirmational holism does not allow the mathematical naturalist to make the inference from the acceptability of the methodology of applied mathematics to the acceptability of the methodology implicit in mathematical practice as a whole. Therefore mathematical naturalism fails to accommodate the methodological autonomy of mathematics within Quinean scientific naturalism.

\author{
Marianna Antonutti Marfori \\ Department of Philosophy \\ University of Bristol \\ BS8 1TB - Bristol, United Kingdom \\ plmam@bristol.ac.uk
}

\title{
References
}

Busch, Jacob. Forthcoming. Can the new indispensability argument be saved from Euclidean rescues? Synthese.

Colyvan, Mark. 2001. The Indispensability of Mathematics. New York: Oxford University Press.

Colyvan, Mark. 2007. Mathematical Recreation Versus Mathematical Knowledge. In Mathematical Knowledge, ed. By M. Leng, A. Paseau, and M. Potter. Oxford: Oxford University Press.

Dieterle, Jill. 1999. Mathematical, astrological, and theological naturalism. Philosophia Mathematica 7:129-135.

Field, Hartry.1980. Science without Numbers: a defense of nominalism. Oxford: Blackwell.

Leng, Mary. 2010. Mathematics and Reality. Oxford: Oxford University Press.

Maddy, Penelope. 1992. Indispensability and Practice. The Journal of Philosophy 89:275-289.

Maddy, Penelope. 1997. Naturalism in Mathematics. Oxford: Oxford University Press.

Maddy, Penelope. 2007. Second Philosophy. A Naturalistic Method. New York: Oxford University Press.

Paseau, Alexander. 2005. Naturalism and the Authority of Philosophy. The British Journal for the Philosophy of Science 56:377-396. 
Quine, Willard Van Orman. 1953. From a Logical Point of View. Cambridge, MA: Harvard University Press.

Quine, Willard Van Orman. 1969. Ontological Relativity and Other Essays. New York: Columbia University Press.

Quine, Willard Van Orman. 1981. Theories and Things. Cambridge, MA: Harvard University Press.

Quine, Willard Van Orman. 1986. Reply to Charles Parsons. In The Philosophy of W.V. Quine, ed. By L. Hahn, and P. Schilpp. Peru, IL: Open Court.

Tennant, Neil. 2000. What Is Naturalism in Mathematics, Really? Philosophia Mathematica 8:316-38.

Tappenden, Jamie. 2001. Recent Work in Philosophy of Mathematics. The Journal of Philosophy 98:488-497.

Weir, Alan. 2005. Naturalism Reconsidered. In The Oxford Handbook of Philosophy of Mathematics and Logic, ed. by S. Shapiro. New York: Oxford University Press. 